\title{
Acid Sensing lon Channels (ASICS) in NS20Y cells - potential role in neuronal differentiation
}

\author{
Zaven O'Bryant ${ }^{\dagger}$, Tiandong Leng ${ }^{\dagger}$, Mingli Liu, Koichi Inoue, Kiara T. Vann and Zhi-gang Xiong ${ }^{*}$ (D)
}

\begin{abstract}
Cultured neuronal cell lines can express properties of mature neurons if properly differentiated. Although the precise mechanisms underlying neuronal differentiation are not fully understood, the expression and activation of ion channels, particularly those of $\mathrm{Ca}^{2+}$-permeable channels, have been suggested to play a role. In this study, we explored the presence and characterized the properties of acid-sensing ion channels (ASICs) in NS2OY cells, a neuronal cell line previously used for the study of neuronal differentiation. In addition, the potential role of ASICS in cell differentiation was explored. Reverse Transcription Polymerase Chain Reaction and Western blot revealed the presence of ASIC1 subunits in these cells. Fast drops of extracellular pH activated transient inward currents which were blocked, in a dose dependent manner, by amiloride, a non-selective ASIC blocker, and by Psalmotoxin-1 (PCTX1), a specific inhibitor for homomeric ASIC1a and heteromeric ASIC1a/2b channels. Incubation of cells with PCTX1 significantly reduced the differentiation of NS2OY cells induced by cPt-cAMP, as evidenced by decreased neurite length, dendritic complexity, decreased expression of functional voltage gated $\mathrm{Na}^{+}$channels. Consistent with ASIC1a inhibition, ASIC1a knockdown with small interference RNA significantly attenuates cpt-cAMP-induced increase of neurite outgrowth. In summary, we described the presence of functional ASICs in NS2OY cells and demonstrate that ASIC1a plays a role in the differentiation of these cells.
\end{abstract}

Keywords: Acid Sensing Ion Channels (ASICS), Neuronal differentiation, Neurite growth, Dendrites, NS20Y

\section{Background}

Neuronal differentiation is essential for the development of the nervous system. A hallmark characteristic of differentiation is the sprouting of neurites which later become axons and dendrites. Major changes in membrane proteins are observed during the differentiation, maturation, and development of neurons, for example increased expression of acid-sensing ion channels (ASICs) [1]. Although the precise mechanisms underlying neuronal differentiation are not fully understood, expression and activation of ion channels, particularly those which are $\mathrm{Ca}^{2+}$-permeable, have been suggested to play an important role in the process [2-4].

ASICs are proton-gated cation channels belonging to the degenerin/epithelial $\mathrm{Na}^{+}$channel (DEG/ENaC)

\footnotetext{
*Correspondence: zxiong@msm.edu

${ }^{\dagger}$ Equal contributors

Neuroscience Institute, Morehouse School of Medicine, 720 Westview Drive

SW, Atlanta, GA 30310-1945, USA
}

superfamily. There are at least four genes that encode six alternatively spliced transcripts: ASIC1a, ASIC1b, ASIC2a, ASIC2b, ASIC3 and ASIC4. ASIC1a, a primary subunit highly expressed in the central and peripheral neurons, is highly sensitive to decrease in extracellular $\mathrm{pH}$ [5]. Studies using knockout mice have suggested that activation of ASIC1a contributes to synaptic plasticity, learning and memory [6]. It is unclear however whether ASICs play any role in neuronal differentiation. In this study, we first explored the presence and characterized the properties of ASICs in NS20Y cells, a neuronal cell line that has been previously used to study neuronal differentiation. Next, we determined whether ASIC inhibition affects the differentiation of these cells. Our data provides strong evidence that functional ASIC1a channels are expressed in NS20Y cells and that activation of these channels may play a role in neuronal differentiation. 


\section{Methods}

\section{Cell culture}

NS20Y cells, derived from the mouse neuroblastoma, were cultured in Dulbecco's Modified Eagle's Medium (Invitrogen), supplemented with 10 \% FBS, 100 units/ml penicillin, and $100 \mu \mathrm{g} / \mathrm{ml}$ streptomycin. Cells were plated at $10-20 \%$ confluence on $35 \mathrm{~mm}$ dishes coated with poly-L-ornithine and maintained at $37{ }^{\circ} \mathrm{C}$ in a humidified incubator with $5 \% \mathrm{CO}_{2}-95 \%$ atmosphere. For differentiation, cells were treated with $1 \mathrm{mM}$ cpt-cAMP for $72 \mathrm{~h}$ or $1 \mathrm{mM}$ cpt-cAMP and $10 \mathrm{nM}$ PcTX1 by adding reagents directly to cell media. The culture medium was not changed during the $72 \mathrm{~h}$ treatment. The $\mathrm{pH}$ during experiments were 7.62 in control, 7.63 in cpt-cAMP, and 7.64 in cpt-cAMP + PcTX1 treated medium.

\section{Evaluation of neuronal differentiation}

Cells in $35 \mathrm{~mm}$ dishes were examined at 400X magnification and photographed using phase contrast microscopy (Nikon). Cells were washed three times with extracellular solution (ECF) before photographs were taken. Neurite length and cell complexity were measured using Nikon Image Software (NIS) (Nikon Instruments, Inc., Melville, NY, USA). For each experiment, at least 5 random fields were selected for evaluation. Number of primary dendrites and total neurite length were quantified [7]. In these experiments neurites are defined as any process that extends from the soma. Neurite length (in $\mu \mathrm{m}$ ) was quantified by using a free hand line tool measuring the distance from the neurite tip to where the neurite joins the soma [7]. Exclusion criteria included: 1) cell clusters typically greater than or equal to two, 2) where the total neurite length cannot be ascertained because neurites extend out of the field of view, 3) neurites that appear to have formed neurite-neurite or neurite-somatic connections, and 4) cases of extensively branched or overlapped neurites [7].

Sholl analysis is a widely used method in neurobiology to quantify the complexity of dendritic arbors [8]. The Sholl analysis of NS20Y cells was conducted by plotting the number of neurite intersections against the radial distance from the soma [7].

\section{Electrophysiology}

Whole cell patch clamp recordings were performed as described previously [9]. Patch pipettes were pulled by a two-step puller (PP83; Narishige, Tokyo, Japan) from thin walled borosilicate glass $(1.5 \mathrm{~mm}$ diameter, World Precision Instruments, Sarasota, FL). The pipettes had a resistance of 3-4 $\mathrm{M} \Omega$ when filled with intracellular solution: 140 mM CsF, 10 mM HEPES, 11 mM EGTA, 2 mM tetraethylammonium chloride, $1 \mathrm{mM} \mathrm{CaCl}_{2}, 2 \mathrm{mM} \mathrm{MgCl}$ and $4 \mathrm{mM}$ MgATP, pH 7.3 (adjusted with $\mathrm{CsOH}$ ), 290-300 mOsm adjusted with sucrose. Extracellular solution contained: $140 \mathrm{mM} \mathrm{NaCl}, 5.4 \mathrm{KCl}, 2 \mathrm{mM} \mathrm{CaCl}_{2}$,
$1 \mathrm{mM} \mathrm{MgCl} 2,10 \mathrm{mM}$ HEPES, $10 \mathrm{mM}$ glucose, $\mathrm{pH}$ 7.4, 320-330 mOsm. When needed, $300 \mathrm{nM}$ tetrodotoxin was used in ECF to block the $\mathrm{Na}^{+}$currents. PcTX1 (Peptide International) was dissolved in $\mathrm{ddH}_{2} \mathrm{O}$ at $20 \mu \mathrm{M}$ before adding to extracellular solutions. Amiloride was dissolved in dimethyl sulfoxide (DMSO) at $100 \mathrm{mM}$ before adding to extracellular solutions to obtain final working concentrations. Tetrakis-(2-Pyridylmethyl) ethylenediamine (TPEN) and Zinc Chloride were dissolved in $\mathrm{ddH}_{2} \mathrm{O}$ before adding to extracellular solutions. Unless described otherwise, all chemicals were purchased from Sigma.

Whole cell patch clamp recordings were done with an Axopatch 200B amplifier and Digidata 1320 DAC unit. Unless indicated otherwise, cells were clamped at a holding potential of $-60 \mathrm{mV}$. ASIC currents were elicited by $\mathrm{pH}$ drops from 7.4 to acidic $\mathrm{pH}$ values as indicated. Currents were activated at least $1 \mathrm{~min}$ apart to achieve a complete recovery from desensitization. A multi-barrel perfusion system (SF-77 Warner Instruments, Hamden, CT) was used to achieve a rapid exchange of extracellular solutions. To generate a current-voltage (I-V) relationship, voltage steps were initiated from -100 and $+100 \mathrm{mV}$ from a holding potential of $-60 \mathrm{mV}$ were applied at $1 \mathrm{~s}$ interval. The data were analyzed with pClamp and Sigma Plot software.

\section{Western blot}

Cells were treated with lysis buffer $(50 \mathrm{mmol} / \mathrm{L}$ Tris- $\mathrm{HCl}$, $150 \mathrm{mmol} / \mathrm{L} \mathrm{NaCl}, 1 \%$ Triton X-100, protease and phosphatase inhibitor cocktail) and collected by scraping into individual aliquots. The samples were put on ice for $30 \mathrm{~min}$, centrifuged at $12,000 \mathrm{~g}$ at $4{ }^{\circ} \mathrm{C}$ for $30 \mathrm{~min}$, and then supernatants were collected. Protein concentration was measured using the Bio-Rad protein assay kit (BioRad, Hercules, CA, USA). Thereafter, the proteins were mixed with Laemmli sample buffer and boiled at $95{ }^{\circ} \mathrm{C}$ for 5 min. Proteins were separated by $10 \%$ SDS-PAGE, followed by electrotransfer to polyvinylidene difluoride membranes. Blots were probed with antibodies against ASIC1 (rabbit anti-mouse/human, 1:1,000; Gift from Dr. Xiangming Zha, University of South Alabama, Mobile, AL, USA) or beta-actin (1:2,000; Abcam, Cambridge, MA, USA), detected using horseradish peroxidase-conjugated secondary antibody (1:1,000; Cell Signaling, Danvers, MA, USA), and visualized by ECL (Amersham Biosciences Piscataway, NJ) and Blue Autoradiography film (MedSupply Partners, Atlanta, GA). The intensity of the protein bands were quantified using NIH Image J software.

Reverse Transcription - Polymerase Chain Reaction (RT-PCR) RT-PCR was used to examine the expression of individual ASIC subunits, as described in our previous studies [10]. Total RNAs were isolated from NS20Y cells with Trizol reagent (Invitrogen), according to the manufacturer's 
protocol. Equal amount of total RNA was reverse transcribed and PCR amplified with Superscript II (Invitrogen) using specific primers for individual ASIC subunit. ASIC1a forward5'-TCCTATGAGCGGCTGTCTCT-3', ASIC1a, reverse 5'-TGCTTTTCATCAGCCATCTG-3', ASIC1b forward $5^{\prime}$-GGCCTTTGTCATAGCACTGGG TGC -3', ASIC1b reverse 5' -TTCCCATACCGCGTGAAGACCAC -3', ASIC2a forward 5'-CGCCAACACCTCTACTCTCC-3', ASIC2a reverse 5' -TGCCATCCTCGCC TGAGTTA-3', ASIC2b forward 5' -CCTTGGCTTGCTG TTGTCCT-3' ASIC2b reverse 5' -TGCCATCCTCGCCTGAGTTA-3', ASIC3 forward 5'-GTCTGGACCCTGCTGAACAT-3', ASIC3 reverse 5'-GGCTCTGGATCAAA GTCGGG-3', ASIC4 forward 5'-GGGCTAGCATCCTCACCTTG-3', ASIC4 reverse (5'-GGCCCAGTTTCA TGGGTACT-3'. RT positive (+) samples was run with the reverse transcriptase, while RT negative (-) samples were run without reverse transcriptase. The RT-PCR products were electrophoresed on $1.5 \%$ agarose gel.

\section{ASIC1a shRNA transfection}

Short hairpin ASIC1a (shASIC1a) and control shRNA were purchased from SuperArray Bioscience Corporation (Frederick, MD), each vector contains the shRNA under control of U1 promoter and the GFP gene, for the enrichment of transiently transfected cells. NS20Y cells were transfected with $5 \mu \mathrm{g}$ of each specific ASIC1a shRNA or control shRNA using Lipofectamine ${ }^{\mathrm{Tm}}$ reagent in serum free OptiMEM-1 medium (Invitrogen, Carlsbad, CA) per $35 \mathrm{~mm}$ dish according to the manufacture's instruction. After transfection, cells were grown for further $72 \mathrm{~h}$ in growth medium as indicated in each experiment before utilization.

\section{Statistical analysis}

Data were expressed as mean \pm SEM. Where applicable, multiple groups were compared using analysis of variance (ANOVA). Two groups were compared using Student's $t$-test (paired and unpaired where appropriate). Values of $p<0.05$ were considered statistically significant.

\section{Results \\ Detection of ASIC transcript and protein in NS20Y cells} Using RT-PCR, the presence of ASIC transcripts in NS20Y cells was investigated. The mRNA expression of all ASIC subunits including ASIC1a, 1b, 2a, 2b, 3 and 4 were examined. RT-PCR results clearly show the presence of ASIC1a and ASIC1b transcripts at the expected sizes (299 bp and $399 \mathrm{bp}$ ) without detection of other subunits (Fig. 1a). We further examined the expression of ASIC1a protein by Western blot. Chinese Hamster Ovarian (CHO) cells and $\mathrm{CHO}$ cells with stable ASIC1a expression (CHO-ASIC1a) were used as negative and positive controls. Western blots showed clear immunoreactivity to the ASIC1a in NS20Y cells at the expected molecular weights (Fig. 1b). Taken together, these findings indicate that ASIC1a is expressed in NS20Y cells.

\section{Characterization of the ASIC currents in NS20Y cells}

Using patch-clamp recording, we then studied the acidactivated currents and examined the effect of various pharmacologic agents known to modulate ASICs. ASIC currents were induced by an extracellular $\mathrm{pH}$ drop from 7.4 to 6.0 [9]. As shown in Fig. 2, drop of extracellular $\mathrm{pH}$ from 7.4 to 6.0 induced transient inward currents. Amiloride, a commonly used nonspecific inhibitor of ASICs [11-14], blocked the acid activated currents in NS20Y cells in a dose-dependent manner with a halfmaximal inhibitory concentration $\left(\mathrm{IC}_{50}\right)$ of $11.04 \mu \mathrm{M}$ $(n=5)$ (Fig. 2a).

Psalmotoxin-1 (PcTX1), isolated from the venom of tarantula Psalmopoeus cambridgei, potently and specifically inhibits the proton-gated currents mediated by homomeric ASIC1a expressed in heterologous systems [15]. In addition, PcTX1 also inhibits the current mediated by heteromeric ASIC1a/2b channels [11]. We tested the effect of PcTX1 on ASIC currents in NS20Y cells. As shown in

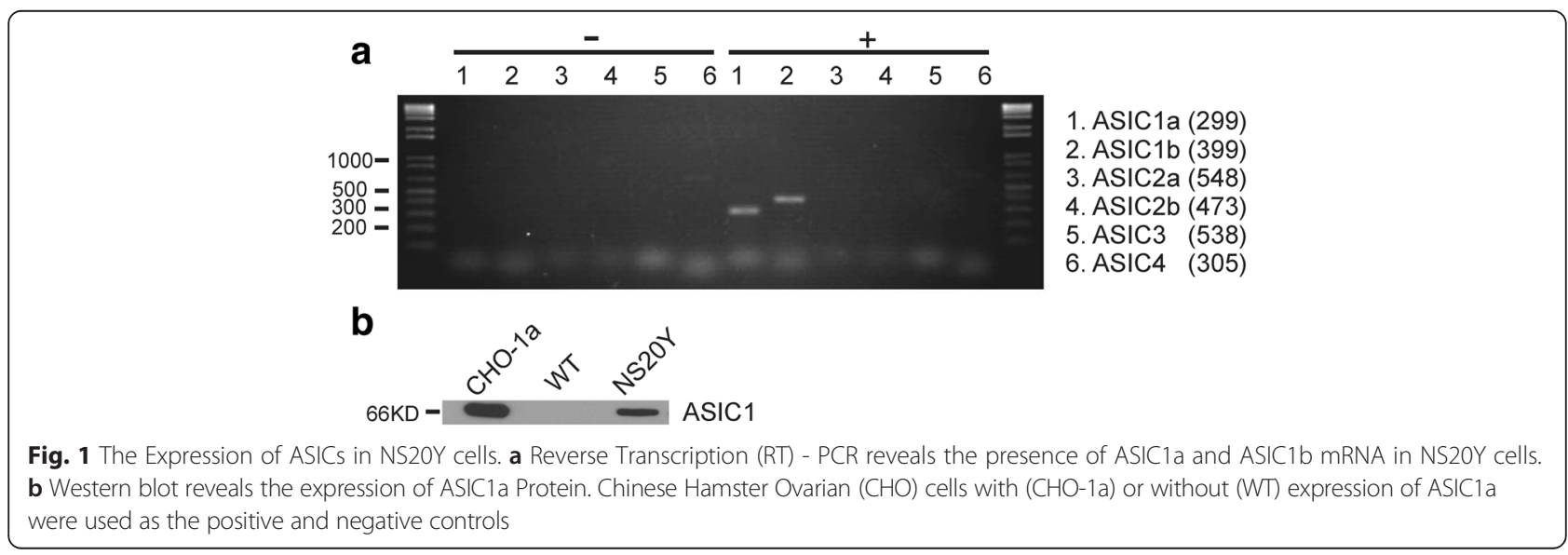


a
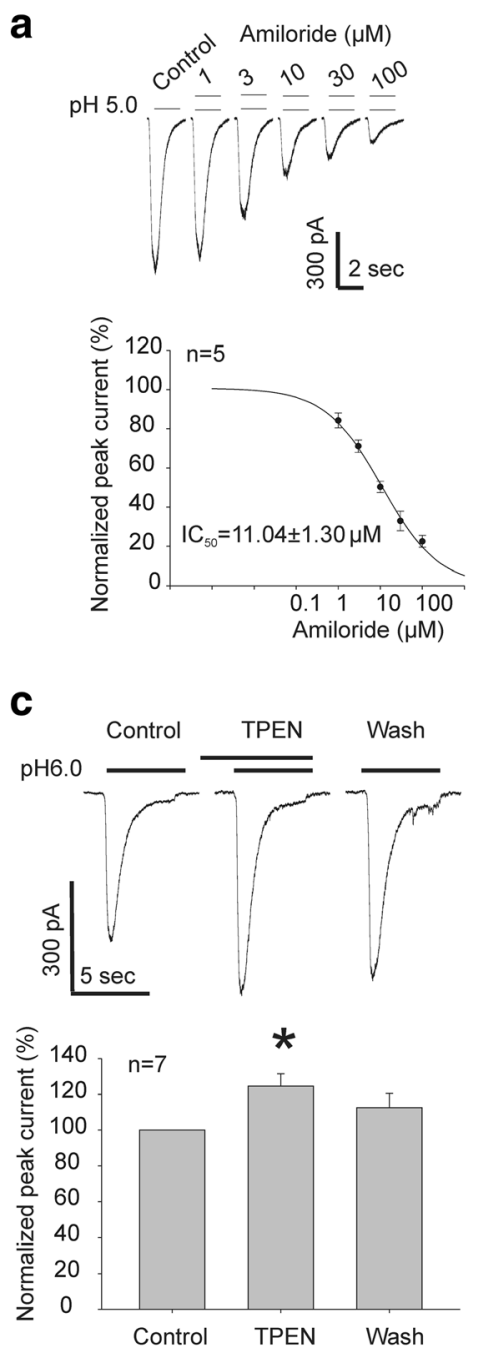

b
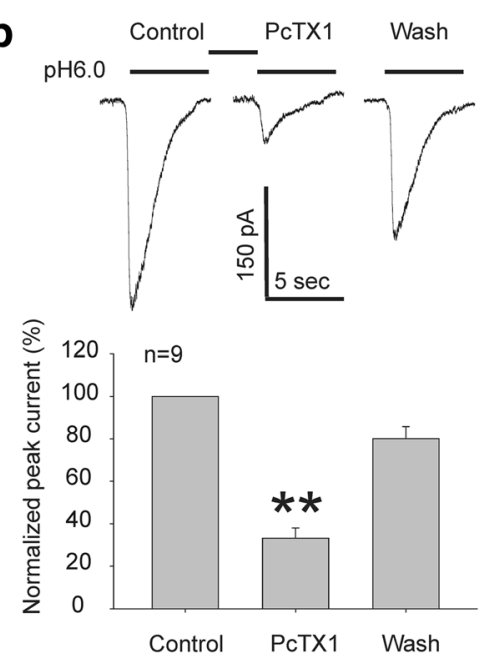

d
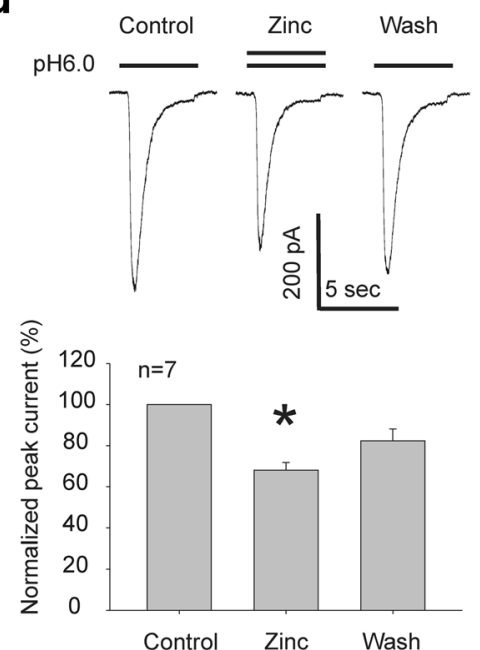

Fig. 2 Inhibition of ASIC current by amiloride and PCTX1 and modulation of ASIC current by zinc and zinc chelator in NS20Y cells. a Amiloride dose dependently inhibits the ASIC current in NS20Y cells with a half-maximal inhibitory concentration $\left(\mathrm{I}_{50}\right)$ of $11.04 \mu \mathrm{M}(n=5)$. b PCTX1, at 10 $\mathrm{nM}$, also significantly inhibits the ASIC current in NS20Y cells $\left({ }^{* *} p<0.01, n=9\right)$. c Zinc chelator, TPEN, at $100 \mu$ M, significantly potentiates ASIC currents $(p<0.05, n=7)$. $\mathbf{d}$ Zinc, $100 \mu \mathrm{M}$ causes a significant inhibition of ASIC currents $(p<0.05, n=7)$

(Fig. 2b), after several minutes of perfusion, PcTX1 (10 $\mathrm{nM})$ produced a significant inhibition of ASIC currents in NS20Y cells $(p<0.05$, paired student's $t$-test). In 9 cells tested, an average inhibition of $66.9 \pm 14.8 \%$ of the current amplitude was achieved. Since our RT-PCR data did not show clear expression of ASIC2b (Fig. 1), this result suggested that the acid-activated current in NS20Y cells is largely mediated by the homomeric ASICla channels.

Zinc modulates ASIC currents, but different ASIC subunit stoichiometry responds differently to zinc; as such, zinc may either potentiate or inhibit the acidactivated currents [16, 17]. For example, at nanomolar concentrations, zinc inhibits ASICla containing channels with an $\mathrm{IC}_{50}$ of $\sim 10 \mathrm{nM}$ [16]. While at high micromolar concentrations, zinc potentiates ASIC2a containing channels [17]. Zinc chelator tetrakis-(2-Pyridylmethyl) ethylenediamine (TPEN) potentiates ASICla current, by removing zinc mediated inhibition. Application of $100 \mu \mathrm{M}$ TPEN significantly increased the amplitude of ASIC currents in NS20Y cells, further supporting the presence of ASICla containing channels $(p<0.01$, paired student's $t$ test, $n=7$, Fig. 2c). We found that addition of $100 \mu \mathrm{M}$ zinc caused no potentiation but an inhibition of ASIC currents in NS20Y cells $(p<0.01$, paired Student's $t$-test, $n=7$, Fig. 2d), which is consistent with the presence of ASIC1a containing channels.

\section{ASIC blockade inhibits neuritogenesis}

To explore the potential role of ASICs in neuronal differentiation, the effect of ASIC inhibition on cpt-cAMP induced neuritogenesis of NS20Y cells was studied. Treatment of NS20Y cells with $1 \mathrm{mM}$ cpt-cAMP for 
72-h has been shown to induce clear differentiation $[18,19]$. Differentiation was analyzed morphologically by extension and branching of neurites, and by directly counting the number of primary dendrites (explained in detail in Methods). While control cells are lack of extensive branches (Fig. 3a, upper panel), cells treated with $1 \mathrm{mM}$ cpt-cAMP for $72 \mathrm{~h}$ show significantly increased neurite number, length, and arborization (Fig. 3a, middle panel), co-incubation of $10 \mathrm{nM}$ PcTX1 with $1 \mathrm{mM}$ cptcAMP for 48 and $72 \mathrm{~h}$ significantly decreased the number of neurite branching and neurite length compared to $1 \mathrm{mM}$ cpt-cAMP treatment alone $(n=264, p<0.05$, Fig. 3a, b, c). PcTX1 alone has no significant effect on neurite growth ( $p=0.38, n=268$, data not shown).
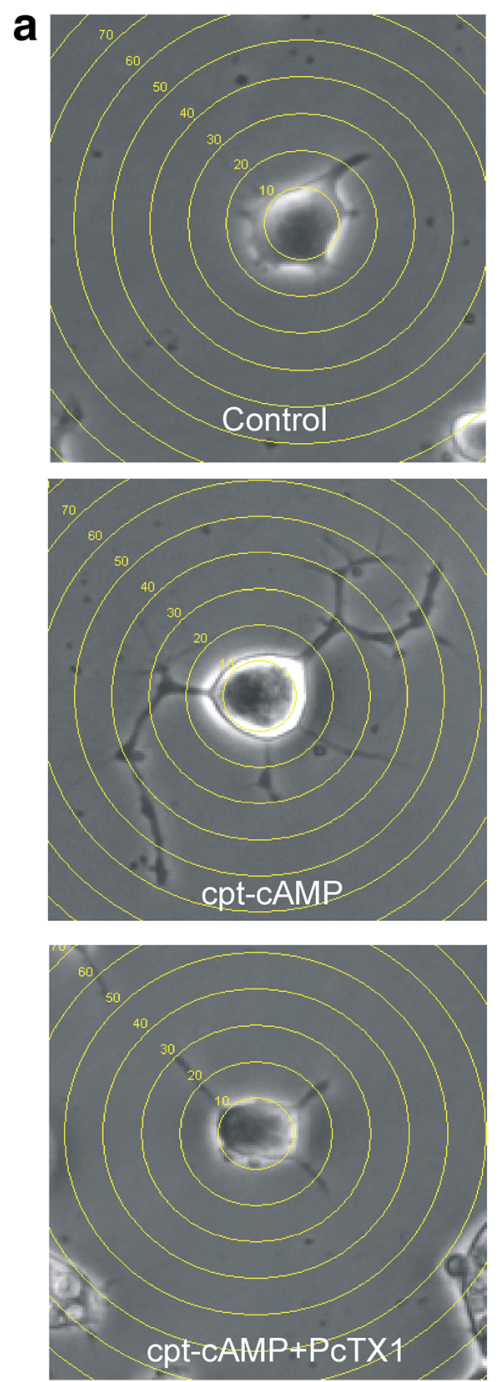
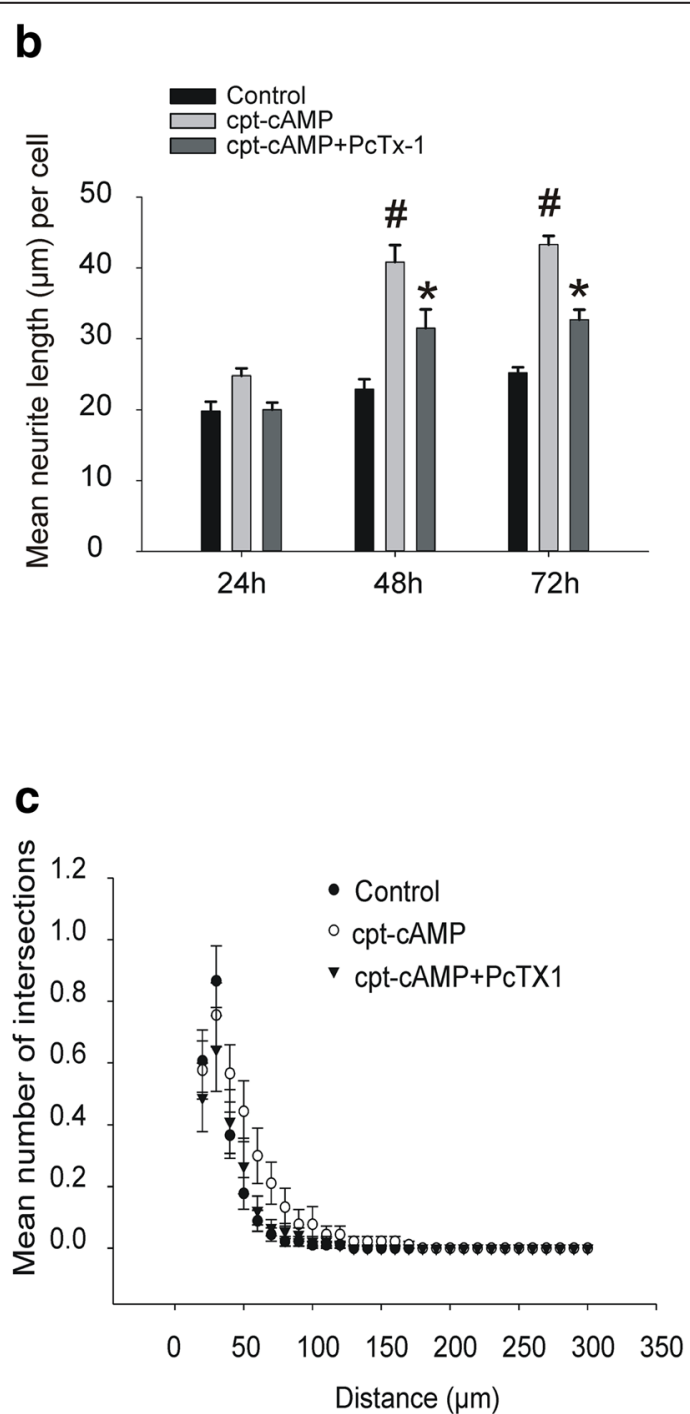

Fig. 3 Effect of PCTX1 on cpt-cAMP induced differentiation of NS20Y cells. a Example images showing morphology of NS20Y cells in different treatment groups. For Sholl analysis of dendritic complexity, concentric rings were placed at the center of the soma radiating outward at a $10 \mu \mathrm{m}$ intervals. Neurite complexity is measured by the number of neurites per intersection. Top panel: Control - shows NS20Y cells grown in media without pharmacologic treatment. Cells display a full, rounded soma with few, short undeveloped processes. Middle panel: cpt-cAMP treated shows NS20Y cells treated with $1 \mathrm{mM}$ cpt-cAMP for 72-h. Cells have large cell bodies, multiple and elongated processes with extensive branching. Lower panel: cpt-cAMP + PcTX1 - shows NS20Y cells treated with $1 \mathrm{mM}$ cpt-cAMP and 10 nM PcTX1 for 72-h. These cells have a reduced neurite extension and processes. Photomicrographs were taken at 400x, phase contrast. b Summary data expressed as the mean neurite length/cell at time points of 24,48 , and $72 \mathrm{~h}$. cpt-cAMP significantly increases the mean length of neurite extension ( ${ }^{2} p<0.05$ vs. control). When PcTX1 is added, there is a significant reduction in neurite length compared to cpt-cAMP treated cells ( $\left.{ }^{*} p<0.05\right)$. c Summary Sholl analysis data of control, cpt-cAMP (1 mM) treated and cpt-cAMP (1 mM) + PcTX1 (10nM) treated cells at $72 \mathrm{~h}$. The plot shows the mean number of neurite intersections at indicated distance from the soma. Treatment with cpt-cAMP significantly increased the dendritic complexity $(p<0.05$ vs. control, two way ANOVA, $n=90$ cells for each group from 3 separate experiments). Co-treatment with 10 nM PCTX1 significantly attenuated the increase of dendritic complexity induced by cpt-cAMP ( $p<0.05$ between cpt-cAMP and cpt-cAMP + PcTX1, two way ANOVA, $n=90$ cells from 3 separate experiments) 
The Sholl analysis method has been used extensively in neuronal cultures for the studies of dendritic complexity [20,21]. We applied this technique in cultures of NS20Y cells (Fig. 3a). Cells treated with $1 \mathrm{mM}$ cptcAMP show neurites that significantly extend from the soma and contain more branches than control cells at distances between $50-90 \mu \mathrm{m}(p<0.05)$ (total of 90 cells measured from 3 independent experiments, two-way ANOVA) (Fig. 3c), while cells co-treated with $1 \mathrm{mM}$ cpt-cAMP and $10 \mathrm{nM}$ PcTX1 have neurites that are not significantly different from control $(p=0.24)$ (total of 90 cells measured from 3 independent experiments) at the same distances.

PcTX1 is generally accepted to be a specific inhibitor of ASIC1a however there are reports that suggest inhibition of ASIC1a/2b heteromeric channels [11, 15]. To provide additional evidence that ASIC1a is involved in ctp-cAMP-induced differentiation of NS20Y cells, we determined whether knocking down the expression of ASIC1a with small hairpin interference RNA (shRNA) has an effect on ctp-cAMP-mediated neurite extension. After transfection with plasmid containing controlshRNA-GFP or ASIC1a-shRNA-GFP, cells were treated with $1 \mathrm{mM}$ ctp-cAMP for $72 \mathrm{~h}$. Transfection efficiency was confirmed by a decrease of ASIC1a expression as determined by Western blot (Fig. 4a). In cells treated with control shRNA, ctp-cAMP treatment was able to induce clear neurite growth (Fig. 4b), as described above. However, in cells transfected with ASIC1a-shRNA, average neurite length was significantly decreased compared to that in cells transfected with control-shRNA (Fig. 4b, $p<0.05, n=25-44)$.

\section{ASIC1a expression and current density were increased in differentiated NS20Y cells}

ASIC protein expression and current density after treatment with cpt-cAMP were also examined. Western blots show an increase in ASIC1a expression after treatment with cpt-cAMP for $72 \mathrm{~h}$ (Fig. 5a, b). $(p<0.05, n=12)$. The whole cell patch-clamp recording shows that treatment with $1 \mathrm{mM}$ cpt-cAMP significantly increases the density of ASIC current $(p<0.05, n=12$, Fig. 5c, d). These findings are consistent with other reports showing that ASIC expression increases with neuronal maturation [1].

\section{Inhibition of ASIC1a reduces the amplitude of voltage gated $\mathrm{Na}^{+}$current}

Voltage gated sodium channels have been known to be exclusive to excitable cells, especially those of neuronal origin [22]. Over the course of neuronal differentiation developing neurons begin to express a wide variety of $\mathrm{Na}^{+}$channels [23]. Here, we found that the amplitude of TTX-sensitive voltage gated $\mathrm{Na}^{+}$current increases with $1 \mathrm{mM}$ cpt-cAMP treatment. The increase of the $\mathrm{Na}^{+}$

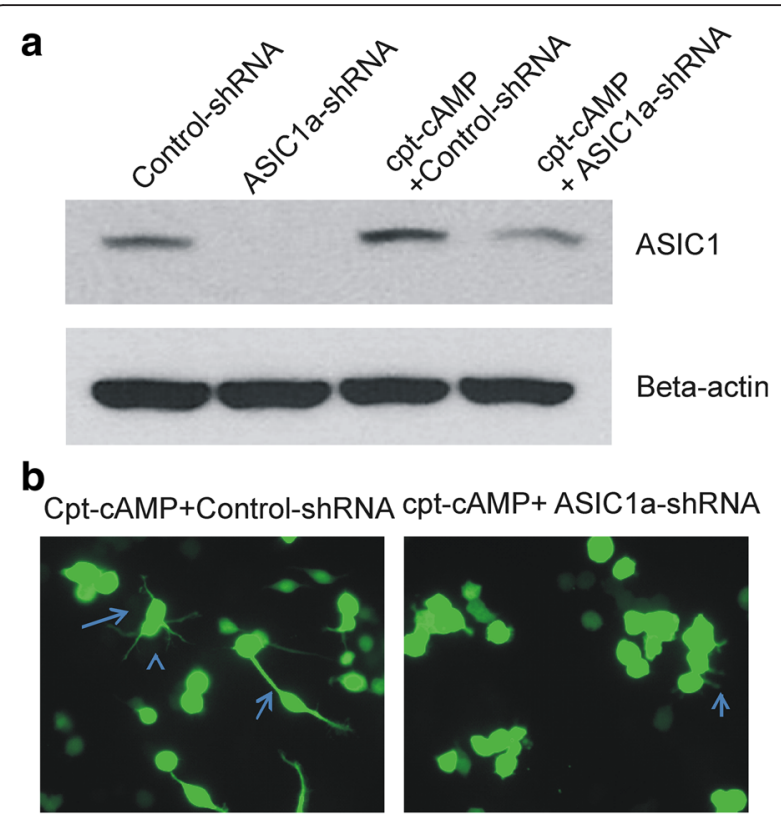

Fig. 4 cpt-CAMP mediated neurite extension is reduced by ASIC1a knock-down. a Western Blot shows that ASIC1a-shRNA treatment for $72 \mathrm{~h}$ significantly decreases ASIC1a expression compared with Control-shRNA treated NS-20Y cells either with or without $1 \mathrm{mM}$ cpt-cAMP treatment. $\mathbf{b}$ Example photomicrographs of cpt-cAMP treated cells in the presence of control-shRNA or ASIC1a-shRNA as indicated. Blue arrows indicated NS20Y cells with enhanced neurite extension. cpt-cAMP + ASIC1a-shRNA treated cells have less and decreased neurite length indicated by the blue arrow. Increases in neurite length seen with application of $1 \mathrm{mM}$ cpt-cAMP in controlshRNA transfected cells is significantly reduced when NS20Y is transfected with ASIC1a-shRNA $(p<0.01)(n=25-44)$

current by cpt-cAMP is however attenuated by cotreatment with $10 \mathrm{nM}$ PcTX1 (Fig. 6a, b, c, d) $(* * p<0.01)$ ( $n=12-14$ cells). The ratios of cells exhibiting the $\mathrm{Na}^{+}$ current are 12/15, 14/14 and 12/14 in control, cpt-cAMP and cpt-cAMP + PcTx-1 treated cells, respectively.

\section{Discussion}

This is the first report, to our knowledge, of the presence of functional ASICs in NS20Y, a mouse neuroblastoma cell line. More importantly, we show that blocking the activity of ASICs inhibits neurite growth/neuronal differentiation. Cyclic-AMP is commonly used to differentiate NS20Y and other clonal cell lines [19, 24]. The use of cyclic-AMPs induces increases in the activities of tyrosine hydroxylase, choline acetyltransferase, the content of poly $(\mathrm{A})^{+}$cytoplasmic RNA, and causes changes in nuclear non-histone proteins $[7,18,25]$. These molecular changes can be tracked by measuring changes in expression of differentially regulated molecules such as neuropeptides [7]. As expected, treatment with cpt-cAMP resulted in an increased neurite extension, dendritic complexity and increase in $\mathrm{Na}^{+}$current. 

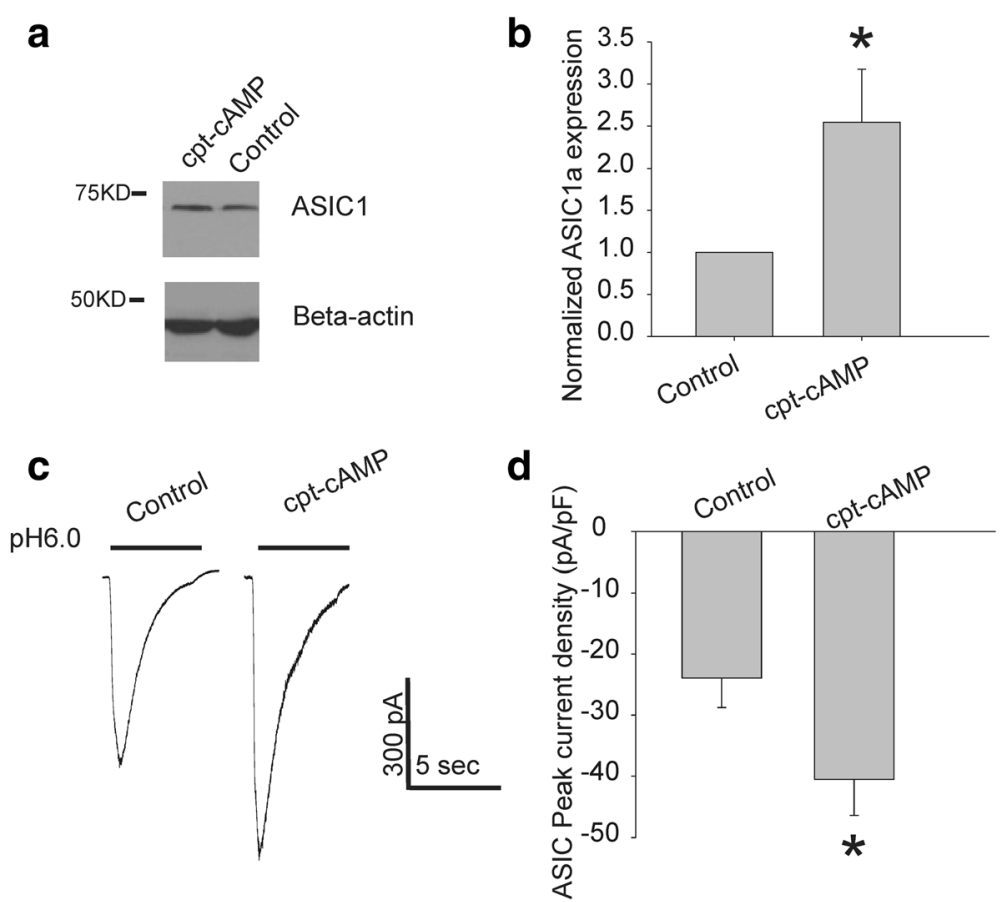

Fig. 5 cpt-CAMP increases ASIC1a expression and ASIC1a current. a ASIC1a protein expression by Western blot. Blots show immunoreactivity to ASIC1 and Beta-actin at their expected molecular weights. After treatment with $1 \mathrm{mM}$ cpt-cAMP, there is an increase in the expression of ASIC1a as evidenced by an increase in density. $\mathbf{b}$ Summary data of Western blotting shows that cpt-CAMP significantly increased ASIC1a protein expression $(p=0.05)(n=12)$. $\mathbf{c}$ Example of whole cell recording that illustrates an increase in ASIC1a current after $72-h$ treatment with cpt-cAMP. $\mathbf{d}$ Summary data showing the ASIC current density in NS2OY cells from different treatment groups. There is a significant increase in the density of peak ASIC current in NS20Y cells treated with cpt-cAMP ( $* 0.05$ vs. control, $n=12$ in each group)

To explore a potential role of ASICs in the differentiation of NS20Y cells, we first determined whether NS20Y cells express ASICs. RT-PCR detected the presence of both ASIC1a and ASIC1b transcripts and Western blot confirmed the presence of ASIC1 protein. The presence of ASIC1a subunit was expected as it is fairly ubiquitous in the central and peripheral neuronal tissues [26-28]. While the presence of ASIC1b in NS20Y cells was surprising, it was not unaccounted for as this cell line has heterogeneous origins, composition, and is mainly found in the peripheral nervous system [14, 24].

Acid-sensing ion channels were further characterized using the whole-cell patch clamp technique. In all cells examined, lowering the extracellular $\mathrm{pH}$ from 7.4 to pH 6.0 evoked a transient inward current at a holding potential of $-60 \mathrm{mV}$. The properties of acid-activated currents in NS20Y cells resemble ASIC currents in cultured primary CNS neurons (human, mouse, and rat) $[9,29,30]$. For example, ASICs in NS20Y were pharmacologically blocked by the non-specific inhibitor amiloride, and the specific inhibitor PcTX1. The concentration response data of amiloride in NS20Y cells is consistent with previously established $\mathrm{IC}_{50}$ for amiloride blockade of ASICs in CNS neurons $[9,30]$. In addition, ASIC currents were inhibited by zinc but potentiated by zinc chelation. Zinc inhibition is consistent with the presence of ASIC1a containing channels [16, 17]. It is plausible that homomeric ASIC1b and heteromeric ASIC1a/1b formations may occur, these configurations of ASICs are not sensitive to PcTX1, which is not the case for the current in NS20Y cells where application of PcTX1 inhibited $70 \%$ of the current. Although PcTX1 also inhibits the current mediated by heteromeric ASIC1a/ ASIC2b channels [11, 15], our RT-PCR result did not show clear expression of $2 \mathrm{~b}$ transcript. Taking together, our data suggest that homomeric ASIC1a channels are predominantly responsible for acid induced currents in NS20Y cells.

Having established the presence of functional ASICs in NS20Y cells, we explored the potential role of these channels in neuronal neuritogenesis. Neuritogenesis was defined as the extension and branching of neurites (length and complexity), similar to other reports in the field $[7,19]$. These parameters were quantified by counting the number of neurites/cell and their length from soma to furthest tip. We noted that while control cells remained small and lacked of extensive branches, cells treated with $1 \mathrm{mM}$ cpt-cAMP increased neurite number, length, overall soma size, and arborization. When PcTX1 was used to inhibit the ASIC1a channels cells treated 


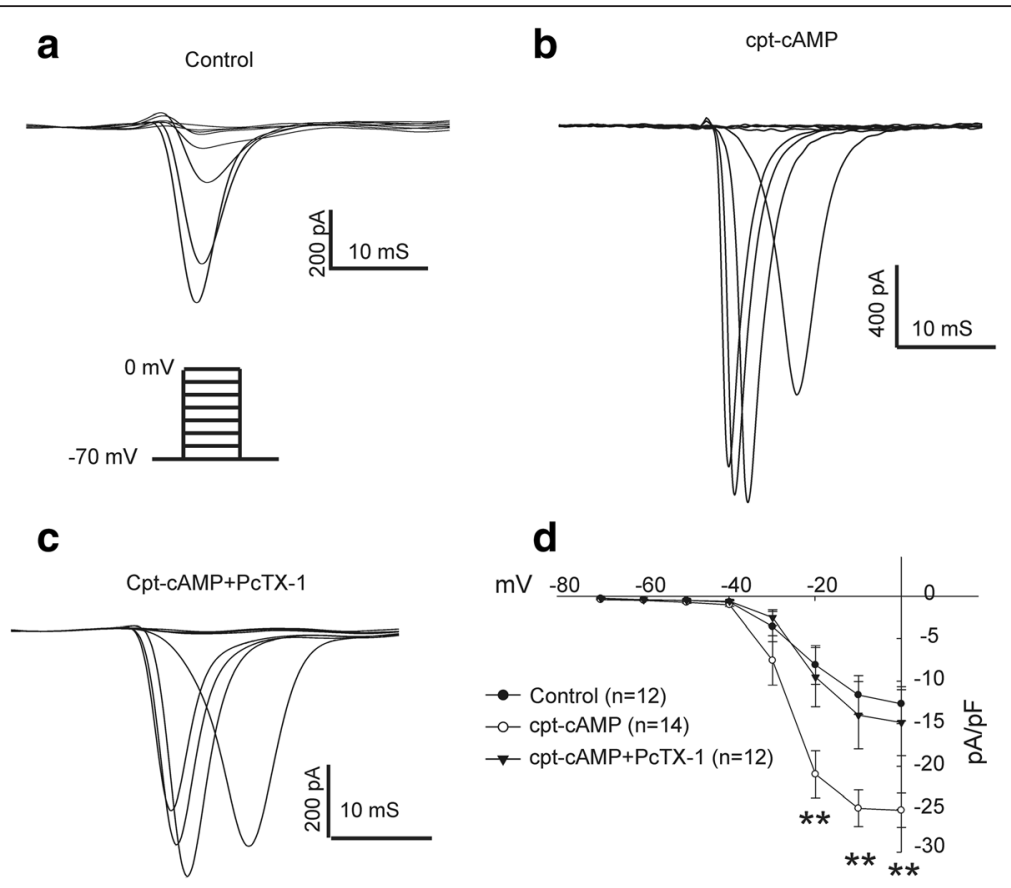

Fig. 6 Inhibition of ASICla attenuates voltage gated $\mathrm{Na}^{+}$current. $\mathbf{a}, \mathbf{b}, \mathbf{c}$ Example traces showing voltage gated $\mathrm{Na}^{+}$currents in NS2OY cells from different treatment groups. Currents were elicited by voltage steps from $-70 \mathrm{mV}$ to $0 \mathrm{mV}$ in $10 \mathrm{mV}$ increments. Treatment of cells with $1 \mathrm{mM}$ cpt-cAMP (b) or 1 mM cpt-cAMP + 10 nM PcTX1 (c) for 72-h causes a concurrent increase or decrease in $\mathrm{Na}^{+}$peak current amplitude, respectively. d Summary I/N plot shows a significant increase in density of voltage gated $\mathrm{Na}^{+}$current after $72-\mathrm{h}$ treatment with $1 \mathrm{mM}$ cpt-cAMP $(* * p<0.01)$, which was largely abolished by $\operatorname{PCTX1}(n=12-14)$

with cpt-cAMP failed to exhibit the same increase of neurite growth. Similarly, knock-down the expression of ASICla resulted in a suppression of cpt-cAMP induced neurite extension. Together, these data suggest that ASIC1a may play an important role in neuronal differentiation of NS20Y cells.

ASIC1a has been suggested to play a role in synaptic plasticity, learning and memory [6], and in acidosismediated cell death $[9,31]$. Since $\mathrm{Ca}^{2+}$ permeability/signaling plays a pervasive role in neuronal maturation, dendritic arborization, and axon outgrowth [18, 32, 33], it is plausible that ASICla activation may play a role in neuritogenesis and neuronal differentiation. ASICs are spatially distributed and co-localize with postsynaptic density protein-95 (PSD-95) at the soma, along dendritic shafts and spines and importantly at the synapses, suggesting the possible involvement of ASIC in normal synaptic transmission and plasticity [6]. Indeed, when ASICs are removed from the synapse, long term potentiation (LTP), the molecular model for learning memory, is impaired [6]. Previous studies have shown that expression of ASICla modulates the density of dendritic spines [34], which may partially explain its role in synaptic plasticity. Our current study suggests that ASIC1a may also play a role in neuronal differentiation and maturation, which may, at least partially, account for its role in synaptic transmission.
NS20Y is a cholinergic cell line which resembles many properties of neurons when differentiated; however, it cannot represent all properties of native neurons and therefore has limitations in neuronal differentiation investigation. Future studies will explore the role of ASIC1a in differentiation/maturation of native neurons.

\section{Abbreviations}

ASICs, acid sensing ion channels; $\mathrm{CHO}$, Chinese Hamster ovarian; CHO-ASIC1a, Chinese Hamster ovarian transfected with ASIC1a; cpt-cAMP, 8 - (4-Chlorophenylthio)-adenosine-3',5'-cyclic monophosphate, sodium salt; $\mathrm{DEG} / \mathrm{ENaC}$, degenerin/epithelial $\mathrm{Na}^{+}$channel; ECF, extracellular fluid; LTP, long term potentiation; PCTX1, psalmotoxin 1; RT-PCR, reverse transcription polymerase chain reaction; TPEN, tetrakis-(2-Pyridylmethyl) ethylenediamine

\section{Acknowledgements}

We thank Dr. An Zhou for the cell line, NS20Y, Dr. Fengxia Yan for assistance in statistical analysis, Dr. Yan Huang and Zhao Zhen for technical assistance.

\section{Funding}

This study was supported by NIH grants R01NS066027, S21MD000101 and U54NS08932 (ZGX), Research Supplements to Promote Diversity (ZO), and Masters of Clinical Research T-32 Training Grant (5T32HL103104-04) (ZO). The funding sources had no input in the design of the study and collection, analysis, and interpretation of data and writing the manuscript.

Availability of data and materials

All data and materials are presented within the article. 


\section{Authors' contributions}

Conceived and designed the experiments: ZO and ZGX; performed experiments: $\mathrm{ZO}, \mathrm{TL}, \mathrm{ML}, \mathrm{KI}$, and KTV; analyzed data: ZO, TL, ML, and ZGX; wrote the paper: ZO, $\mathrm{TL}$, and ZGX. All authors read and approved the final manuscript.

\section{Competing interests}

The authors declare that they have no competing interests.

Received: 27 January 2016 Accepted: 15 June 2016

Published online: 24 June 2016

\section{References}

1. Li M, Kratzer E, Inoue K, Simon RP, Xiong Z-G. Developmental change in the electrophysiological and pharmacological properties of acid-sensing ion channels in CNS neurons. J Physiol. 2010;588:3883-900.

2. Gu X, Olson EC, Spitzer NC. Spontaneous neuronal calcium spikes and waves during early differentiation. J Neurosci. 1994;14:6325-35.

3. Holliday J, Spitzer NC. Spontaneous calcium influx and its roles in differentiation of spinal neurons in culture. Dev Biol. 1990;141:13-23.

4. Rosenberg SS, Spitzer NC. Calcium signaling in neuronal development. Cold Spring Harb Perspect Biol. 2011;3.

5. Xiong ZG, Pignataro G, Li M, Chang SY, Simon RP. Acid-sensing ion channels (ASICs) as pharmacological targets for neurodegenerative diseases. Curr Opin Pharmacol. 2008:8:25-32.

6. Wemmie JA, Chen J, Askwith CC, Hruska-Hageman AM, Price MP, Nolan BC, et al. The acid-activated ion channel ASIC contributes to synaptic plasticity, learning, and.

7. Saito Y, Maruyama K, Saido TC, Kawashima S. Overexpression of a neuropeptide nociceptin/orphanin FQ precursor gene, N23K/N27K, induces neurite outgrowth in mouse NS20Y cells. J Neurosci Res. 1997:48:397-406.

8. Sholl DA. Dendritic organization in the neurons of the visual and motor cortices of the cat. J Anat. 1953;87:387-406.

9. Xiong ZG, Zhu XM, Chu XP, Minami M, Hey J, Wei WL, et al. Neuroprotection in ischemia: blocking calcium-permeable acid-sensing ion channels. Cell. 2004; 118:687-98

10. Li MH, Liu SQ, Inoue K, Lan J, Simon RP, Xiong ZG. Acid-sensing ion channels in mouse olfactory bulb M/T neurons. J Gen Physiol. 2014;143:719-31.

11. Sherwood TW, Lee KG, Gormley MG, Askwith CC. Heteromeric Acid-Sensing Ion Channels (ASICs) composed of ASIC2b and ASICla display novel channel properties and contribute to acidosis-induced neuronal death. J Neurosci. 2011;31:9723-34.

12. Waldmann R, Lazdunski M. $H(+)$-gated cation channels: neuronal acid sensors in the NaC/DEG family of ion channels. Curr Opin Neurobiol. 1998;8:418-24.

13. Benson CJ, Eckert SP, McCleskey EW. Acid-evoked currents in cardiac sensory neurons: a possible mediator of myocardial ischemic sensation. Circ Res. 1999:84:921-8.

14. Chen CC, England S, Akopian AN, Wood JN. A sensory neuron-specific, proton-gated ion channel. Proc Natl Acad Sci. 1998;95:10240-5.

15. Salinas M, Rash LD, Baron A, Lambeau G, Escoubas P, Lazdunski M. The receptor site of the spider toxin PCTX1 on the proton-gated cation channel ASIC1a. J Physiol. 2012;570:339-54.

16. Chu XP, Wemmie JA, Wang WZ, Zhu XM, Saugstad JA, Price MP, et al Subunit-dependent high-affinity zinc inhibition of acid-sensing ion channels. J Neurosci. 2004;24:8678-89.

17. Baron A, Schaefer L, Lingueglia E, Champigny G, Lazdunski M. Zn2+ and H+ Are coactivators of acid-sensing ion channels. J Biol Chem. 2001;276:35367.

18. Saito Y, Maruyama K, Kawano H, Hagino-Yamagishi K, Kawamura K, Saido TC, et al. Molecular cloning and characterization of a novel form of neuropeptide gene as a developmentally regulated molecule. J Biol Chem. 1996;271:15615-22.

19. Sirianni MJ, Fujimoto KI, Nelson CS, Pellegrino MJ, Allen RG. Cyclic AMP analogs induce synthesis, processing, and secretion of prepro nociceptin/ orphanin FQ-derived peptides by NS2OY neuroblastoma cells. DNA Cell Biol. 1999;18:51-8.

20. Gavalda N, Gutierrez H, Davies AM. Developmental switch in NF-KB signalling required for neurite growth. Development. 2009;136:3405-12.

21. Williams PA, Votruba M, Oliphant H, Frizzati A, Littlewood E, Votruba M, et al. Retinal ganglion cell dendritic degeneration in a mouse model of Alzheimer's disease. Neurobiol Aging. 2014.

22. Marban E, Yamagishi T, Tomaselli GF. Structure and function of voltage-gated sodium channels. J Physiol. 1998:508:647-57.
23. Renganathan M, Cummins TR, Waxman SG. Contribution of Nav1.8 sodium channels to action potential electrogenesis in DRG neurons. J Neurophysiol. 2001;86:629-40

24. Amano T, Richelson $E_{1}$ Nirenberg M. Neurotransmitter synthesis by neuroblastoma clones. Proc Natl Acad Sci U S A. 1972;69:258-63.

25. Grouse D, Schrier K, Letendre H, Zubari Y, Nelson G. Neuroblastoma differentiation involves both the disappearance of old and the appearance of new poly(A)' messenger RNA sequences in polyribosomes. J Biol Chem. 1980:255:3871-7.

26. Alvarez de la Rosa D, Zhang P, Shao D, White F, Canessa CM. Functional implications of the localization and activity of acid-sensitive channels in rat peripheral nervous system. Proc Natl Acad Sci U S A. 2002;99:2326-31.

27. Garcia-Anoveros J, Derfler B, Neville-Golden J, Hyman BT, Corey DP. BNaC and $\mathrm{BNaC2}$ constitute a new family of human neuronal sodium channels related to degenerins and epithelial sodiumâ€\%ochannels. Proc Natl Acad Sci. 1997;94:1459-64

28. Waldmann R, Champigny G, Bassilana F, Heurteaux C, Lazdunski M. A protongated cation channel involved in acid-sensing. Nature. 1997;386:173-7.

29. Gao J, $W u L J, X u L, X u T L$. Properties of the proton-evoked currents and their modulation by $\mathrm{Ca} 2+$ and $\mathrm{Zn} 2+$ in the acutely dissociated hippocampus CA1 neurons. Brain Res. 2004;1017:197-207.

30. Li M, Inoue K, Branigan D, Kratzer E, Hansen JC, Chen JW, et al. Acid-sensing ion channels in acidosis-induced injury of human brain neurons. J Cereb Blood Flow Metab. 2010:30:1247-60.

31. Yermolaieva O, Leonard AS, Schnizler MK, Abboud FM, Welsh MJ. Extracellular acidosis increases neuronal cell calcium by activating acid-sensing ion channel 1a. Proc Natl Acad Sci. 2003;101:6752-7.

32. Rosenberg SS, Spitzer NC. Calcium signaling in neuronal development. PLoS One. 2011;6.

33. Saito Y, Maruyama K, Saido TC, Kawashima S. N23K, a gene transiently up-regulated during neural differentiation, encodes a precursor protein for a newly identified neuropeptide nociceptin. Biochem Biophys Res Commun. 1995:217:539-45.

34. Xm Z, Wemmie JA, Green SH, Welsh MJ. Acid-sensing ion channel 1a is a postsynaptic proton receptor that affects the density of dendritic spines. Proc Natl Acad Sci. 2006;103:16556-61.

\section{Submit your next manuscript to BioMed Central and we will help you at every step:}

- We accept pre-submission inquiries

- Our selector tool helps you to find the most relevant journal

- We provide round the clock customer support

- Convenient online submission

- Thorough peer review

- Inclusion in PubMed and all major indexing services

- Maximum visibility for your research

Submit your manuscript at www.biomedcentral.com/submit
Biomed Central 